\title{
APLICAÇÃO DA FERRAMENTA SERVQUAL: A INFLUÊNCIA DO PERFIL DO CLIENTE NA PERCEPÇÃO DA QUALIDADE
}

\section{SERVQUAL TOOL APPLICATION: CUSTOMER PROFILE INFLUENCE ON THE QUALITY OF PERCEPTION}

\author{
Luana Moraes Silva ${ }^{1}$; Rodrigo Bíscaro Nogueira ${ }^{2}$; João Marcos Oliveira ${ }^{3}$; Christiano Luna Arraes ${ }^{4}$ \\ ${ }^{1}$ Universidade Federal do Amazonas - UFAM - Manaus - AM - Brasil \\ luanamoraes.ep@gmail.com \\ ${ }^{2}$ Universidade Federal do Amazonas - UFAM - Manaus - AM - Brasil \\ rodrigobiscaro@gmail.com \\ ${ }^{3}$ Universidade Federal do Amazonas - UFAM - Manaus - AM - Brasil \\ jmoprofe@gmail.com \\ ${ }^{4}$ Universidade Federal do Amazonas - UFAM - Manaus - AM - Brasil \\ chistianoarraes@yahoo.com.br
}

\begin{abstract}
Resumo
O trabalho aborda a análise e a quantificação da satisfação dos clientes de três panificadoras do município de Itacoatiara-AM em relação à qualidade dos serviços prestados, identificando as dimensões da qualidade e os principais problemas na visão dos clientes. A ferramenta utilizada para o planejamento da aplicação, análise e tratamento dos dados foi o SERVQUAL, proposta inicialmente por Parasuraman, Zeithaml e Berry. Para isso, aplicou-se um questionário com 24 questões (22 parâmetros da qualidade e duas perguntas sobre idade e escolaridade) em amostras aleatórias em um total de 416 questionários respondidos pelos clientes das panificadoras. Além da aplicação do SERVQUAL, analisou-se também a influência dos parâmetros da qualidade nos serviços em relação à faixa etária e ao nível de escolaridade dos clientes. $O$ trabalho permitiu analisar os GAP entre as percepções e as expectativas dos clientes, dentre os quais, a dimensão da qualidade tangibilidade (acessibilidade do estacionamento) apresentou o menor valor, ou seja, maior insatisfação dos clientes em relação ao esperado na prestação dos serviços. Pôde-se também identificar diferenças significativas nos GAP devido à faixa etária e a escolaridade dos clientes das três panificadoras. Para análise da confiabilidade do questionário foi utilizado o coeficiente Alfa de Cronbach. O estudo identificou também os pontos críticos relacionados à qualidade nos serviços e permitiu discutir a influência do perfil dos clientes nos resultados obtidos com a aplicação do SERVQUAL, possibilitando propor melhorias para os empreendimentos através de análises mais criteriosas dos resultados.
\end{abstract}

Palavras-chave: SERVQUAL; qualidade em serviços; panificadoras.

\section{Introdução}

O aumento da competitividade acirra a disputa das empresas por uma parcela do mercado. Tornando essencial a busca pela excelência na qualidade tanto nos serviços quanto dos produtos. A qualidade dos serviços constitui uma variável de valor significativo no processo de compra de um produto. Johnston e Clark (2009) conceituam qualidade em serviços como o grau de ajustamento 
entre as expectativas de um cliente e as percepções de um serviço. A compreensão das percepções e expectativas dos consumidores é de fundamental importância para as empresas. Conhecendo as expectativas dos consumidores, a organização oferecerá seus produtos de acordo com a demanda possibilitando maior rentabilidade para o seu negócio (PELISSARI, 2011). Nesse contexto, a competitividade revela-se relevante para a sobrevivência das organizações, as quais investem crescentemente na qualidade dos serviços a fim de se adequar às exigências do mercado. Sendo assim, a qualidade configura-se como importante estratégia no meio empresarial (LACERDA, OLIVEIRA E WISLER, 2013).

Slack et al (2008), define qualidade como a conformidade consistente com as expectativas dos clientes. Nesse âmbito, a qualidade surge como uma adequação aos anseios dos clientes. Batalha (2008) entende que qualidade é o conjunto de aspectos de desempenho valorizados pelo cliente, nos quais a organização focalizará seus esforços. Desse modo, qualidade surge como um conjunto de esforços empreendidos de modo a obter a satisfação dos clientes.

Para Chase (2006), as especificações da qualidade de um produto ou serviço derivam das decisões e das ações tomadas em relação à qualidade de seu projeto e de sua conformidade. $\mathrm{O}$ planejamento e controle da qualidade auxiliam no processo de manutenção contínua da qualidade.

Em uma abordagem baseada no usuário, define-se qualidade como uma variável subjetiva, pois está associada à capacidade de satisfazer os desejos e as necessidades do consumidor (BATALHA, 2008). O conceito de qualidade é aplicado tanto para os produtos quanto para os serviços. A qualidade em serviços apresenta-se como uma maneira de alcançar o sucesso entre os serviços dos concorrentes (SILVA; KOVALESKI; GAIA, 2011). O mercado de serviços apresentase disposto nas mais diversas áreas, dentre elas, os serviços de panificação.

Segundo o Programa de Desenvolvimento da Alimentação, Confeitaria e Panificação (PROPAN, 2014), o setor movimentou R\$ 76,405 bilhões em 2013 e gerou mais de 850 mil empregos diretos e 1,85 milhão indiretos. Objetivando-se analisar esse setor, ainda pouco explorado sob a ótica da qualidade em serviços na perspectiva do cliente, abordou-se a qualidade nos serviços de panificação em três panificadoras no município de Itacoatiara - AM, buscando proporcionar aos empresários locais informações relevantes para a busca de melhorias em seus estabelecimentos, por meio de medidas capazes de atender as exigências dos clientes.

\subsection{Qualidade em serviços}

Segundo o IBGE (2014), o setor de serviços é responsável por 58\% do PIB de ItacoatiaraAM, demonstrando a importância do setor e da qualidade envolvida na prestação dos serviços para o município. Diante deste panorama, a compreenção de qualidade nos serviços, sua extrema amplitude e complexidade e as formas de analisá-la se fazem necessárias. Para Guarienti et al 
(2006), serviços são intangíveis, perecíveis, além disso são produzidos e consumidos simultaneamente. Nesse sentido, a qualidade dos serviços deve visar o máximo de conforto, maior conveniência, mínimo custo, maior justiça e respeito, maior rapidez - entre outros fatores que reflitam a preocupação com a forma de atender às necessidades do seu público cliente, criando, assim, uma nova imagem da organização (GONÇALVES E OLIVEIRA, 2007).

O cliente surge como o avaliador da qualidade do serviço, determinando o sucesso da empresa em longo prazo (JOHNSTON E CLARK, 2009). A compreensão de como se dá o processo de formação da satisfação do cliente apresenta-se como um ponto importante a ser considerado, em que a o nível de satisfação é dado pela relação exixtente entre a expectativa do cliente e a percepção do serviço que foi entregue pelo prestador.

Pisoni (2013) afirma que os consumidores avaliam a qualidade dos serviços quando se referem a preços, instalações físicas e toda e qualquer evidência física que esteja ao alcance da percepção como instalações, equipamentos, bens facilitadores e outros.

A qualidade em serviços é um importante diferencial e deve ser avaliado constantemente, por isso o prestador de serviços deve sempre estar atento se a sua clientela está satisfeita com o atendimento recebido, comparando as expectativas dos clientes com a avaliação do serviço oferecido. A opinião do cliente contribui significativamente para revelar a medida certa da qualidade dos produtos e serviços (BARRETO; SANTOS; SILVA, 2012).

Correa (2009) afirma que as ferramentas de avaliação da qualidade em serviços podem apresentar erros como: assimetria de escalas Likert, escala não elaborada adequadamente, o que pode induzir as respostas, falha do entendimento do cliente entrevistado e a quarta falha refere-se à garantia de que o respondente representa um papel importante na aquisição dos dados para as pesquisas. $\mathrm{O}$ fato de não assumir representatividade da amostra, excesso de preciosismo e o fato de não perguntar se o cliente tem intenção de voltar ou de recomendar o serviço a outras pessoas representam, respectivamente, as falhas de números cinco, seis e sete apontadas pelo autor.

Uma abordagem padrão para se avaliar a qualidade nos serviços consiste em medir a lacuna entre o que os clientes esperam e suas perspectivas em relação ao serviço fornecido (CHASE, 2006). Esta abordagem pode ser medida através do questionário denominado SERVQUAL. No qual, para que haja percepção do serviço é necessário que o cliente tenha necesidades pessoais, experiência passada ou conhecimentos por meio de outras pessoas. As dimensões da qualidade do serviço estão inseridas no conceito de serviço esperado e serviço percebido. A diferença entre as perspactivas e percepções constituem o conceito da qualidade percebida. 


\subsection{SERVQUAL}

Dentre as inúmeras ferramentas da qualidade que estudam os serviços, tem-se a ferramenta SERVQUAL, que pode ser aplicada nos mais variados tipos de empresas prestadoras de serviços. Essa ferramenta consiste de um questionário contendo duas declarações afirmativas, fazendo referência à expectativa do cliente e à sua percepção da qualidade do serviço prestado.

$\mathrm{Na}$ avaliação da qualidade em serviços, técnicas como aplicação de questionários são bastante utilizadas. Guarienti et al (2006), apoiado na metodologia proposta por três autores americanos, Parasuraman, Zeithaml e Berry, afirma que, na Escala SERVQUAL, a qualidade é avaliada pelo consumidor na comparação entre suas expectativas e percepções. Essa ferramenta consiste em um questionário de duas declarações afirmativas, fazendo referência à expectativa do cliente e à sua percepção da qualidade do serviço prestado. O questionário é respondido pelos clientes, avaliando-se as perguntas, e determinando um valor dentro da escala que vai de 01 à 07 (sete) pontos, variando de "discordo totalmente" a "concordo totalmente". A área de aplicação da ferramenta pode ser adaptada ao serviço que se pretende analisar, atendendo a diversos setores de serviços.

Para a análise dos GAP da qualidade, Zeithaml, Parasuraman e Berry desenvolveram o modelo de qualidade de serviços destinado à análise das fontes dos problemas da qualidade e auxílio das empresas prestadoras de serviço (PRASS, SANT'ANNA E GODOY, 2010). Este modelo contém cinco parâmetros dos quais, quatro (GAP 1, GAP 2, GAP 3 e GAP 4) avaliam sob a óptica das empresas de serviço e uma (GAP 5) analisa segundo a percepção dos clientes, o serviço esperado e percebido, conforme ilustra a Figura 1. Com relação ao desempenho do serviço prestado sob a óptica dos clientes, a diferença dos valores obtidos na escala SERVQUAL entre o desempenho do serviço (Percepção do cliente: P) e a Expectativa do cliente (E) é chamada de GAP $=\mathrm{P}-\mathrm{E}$, e é fator fundamental para análise da qualidade nos serviços (GONÇALVES; BELDERRAIN, 2012).

Esse trabalho foi focado na análise do GAP 5, pois o interesse da pesquisa estava direcionada às perspectivas dos clientes em relação à qualidade desempenhada nos estabelecimentos de panificação. Assim, buscou-se estudar as formas de interação das panificadoras com seus clientes através da qualidade na prestação de serviços.

Com relação ao desempenho do serviço prestado sob a óptica dos clientes, a diferença dos valores obtidos na escala SERVQUAL entre o desempenho do serviço (percepção do cliente: P) e a expectativa do cliente (E) é chamada de GAP $=\mathrm{P}-\mathrm{E}$, e é fator fundamental para análise da qualidade nos serviços (GONÇALVES; BELDERRAIN, 2012). 
Aplicação do parametro GAP 5 apresenta ainda cinco dimensões da qualidade dispostas em um certo número de questões. As dimensões da qualidade são: tangibilidade, confiabilidade, responsividade/presteza, empatia e garantia.

Figura 1: Modelo de qualidade de serviços

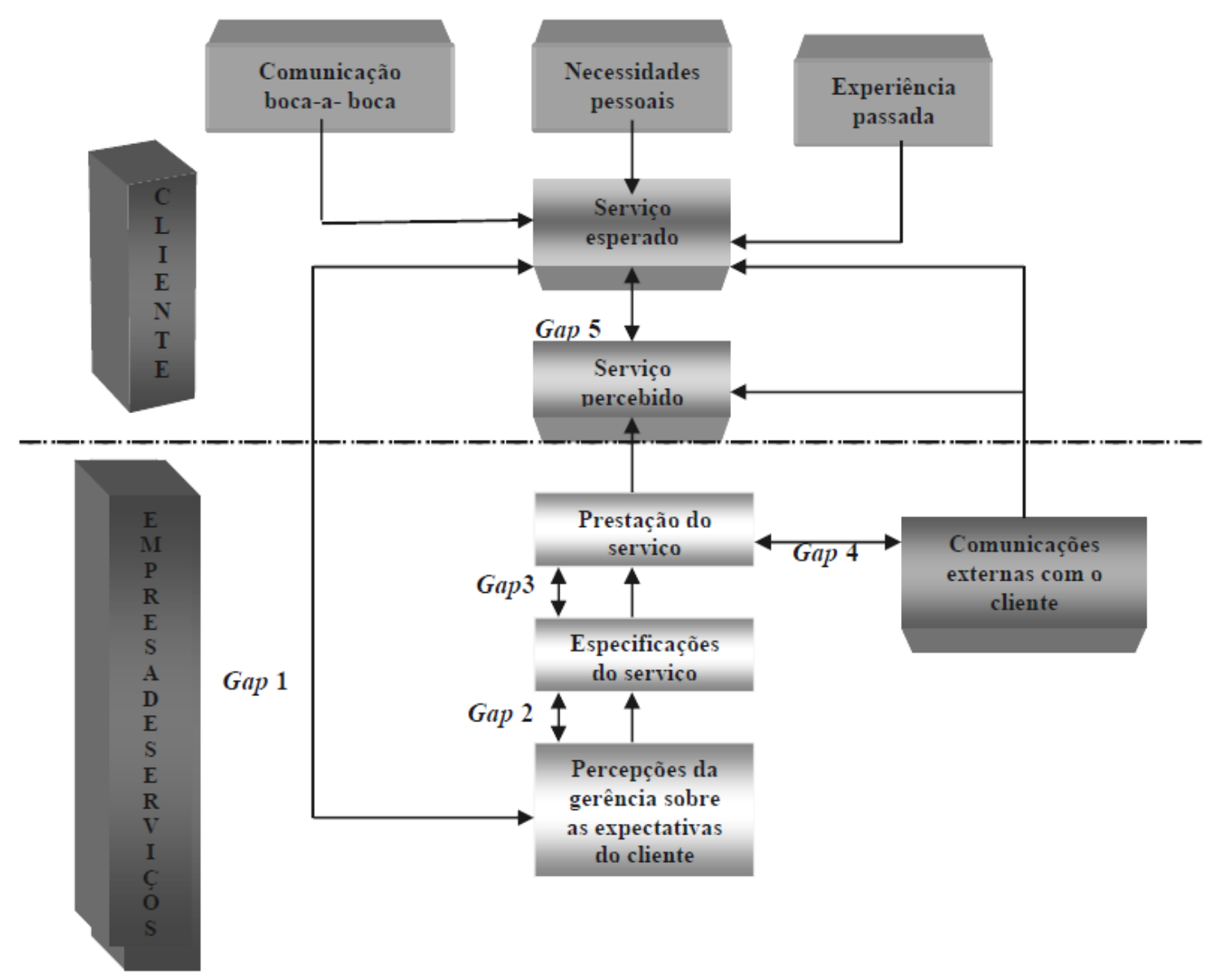

Fonte: PRASS; SANT'ANNA; GODOY (2010)

\subsection{Confiabilidade do questionário}

Almeida, Santos e Costa (2010) definiram a confiabilidade como o grau em que o resultado medido reflete o resultado verdadeiro, ou seja, quanto uma medida está livre da variância dos erros aleatórios. Um instrumento que avalia a confiabilidade é o coeficiente Alfa de Cronbach. Esse coeficiente é definido como uma medida que avalia a confiabilidade para um conjunto de dois ou mais indicadores de constructo e pode ser utilizada para avaliar a confiabilidade do questionário SERVIQUAL (MATTHIENSEN, 2011).

\section{Metodologia}


Este trabalho possui abordagem quali-quantitativa de natureza exploratória utilizando a coleta de dados por meio de questionários. $\mathrm{O}$ trabalho foi executado em quatro etapas principais: (i) identificação das panificadoras objetos de análise e definição do tamanho das amostras; (ii) elaboração dos questionários; (iii) aplicação da escala SERVQUAL e (iv) análise dos resultados encontrados.

(i) Identificação das panificadoras e definição do tamanho das amostras:

Os dados foram coletados das três maiores panificadoras do município de Itacoatiara-AM. As panificadoras foram identificadas como sendo: A, B e C. Os estabelecimentos A e B possuem estrutura mais moderna e estão localizadas no centro da cidade, enquanto o $\mathrm{C}$ está localizado em bairro e possui estrutura mais modesta.

O tamanho da amostra adotado possui intervalo de confiança de $90 \%$ e uma margem de erro mínima de $10 \%$, a aproximação do tamanho da amostra foi calculada pela Equação 1, estimando-se o tamanho da amostra (n) pela Equação 2.

$n_{0}=\frac{1}{E_{0}^{2}}$

Em que, $E_{0}$ representa o erro estimado / tolerável para a pesquisa e $n_{0}$ a aproximação do tamanho da amostra.

$n=\frac{N \cdot n_{0}}{N+n_{0}}$

As variáveis $n, \mathrm{~N}$ e $n_{0}$ representam, respectivamente, o tamanho da amostra, o número total de habitantes da população e aproximação do tamanho da amostra.

Encontrou-se o tamanho mínimo da amostra de 100 entrevistas para o intervalo de $90 \%$. Os dados coletados foram: para a panificadora A obteve-se 140 questionários respondidos, para a panificadora $\mathrm{B}, 141$ questionários e para a panificadora $\mathrm{C}$ o tamanho da amostra foi de 135 questionários respondidos.

(ii) Elaboração dos questionários:

Em seguida foi feito o questionário SERVQUAL, adaptado da metodologia proposta inicialmente por Parasuraman, Zeithaml e Berry (BARRETO et al, 2012), para aplicação em estabelecimentos de panificação. O questionário elaborado como as cinco dimensões da qualidade e com os 22 parâmetros da qualidade está apresentado na Tabela 1.

As perguntas foram organizadas nas cinco dimensões da qualidade (Tangibilidade, Confiabilidade, Presteza, Garantia e Empatia). Onde a primeira dimensão da qualidade (Tangibilidade) compreende as questões de 1 à 4 , a Confiabilidade compreende as questões de 5 à 
9, a Presteza as questões de 10 à 13, a Garantia de 14 à 17 e a última dimensão da qualidade compreende as questões de 18 à 22 .

Os vinte e dois parâmetros da qualidade foram apresentados no formato de perguntas, as quais poderiam ser obtidas sete respostas possíveis, atribuindo pesos de 1 a 7 . As respostas possíveis com os respectivos pesos foram: muito irrelevante (peso 1), irrelevante (peso 2 ou 3), indiferente (peso 4), relevante (peso 5 ou 6) e muito relevante (peso 7).

Também foram solicitadas informações como idade e escolaridade, para relacionar a influencia desses parâmetros na percepção da qualidade. Os dados foram preenchidos na planilha de dados pelos avaliadores da pesquisa de acordo com os valores fornecidos pelos clientes.

Tabela 1: Questionário Servqual

\begin{tabular}{|c|c|c|c|}
\hline $\mathbf{N}^{\circ}$ & & EXPECTATIVA & PERCEPÇÃO \\
\hline 1 & \multirow{4}{*}{ 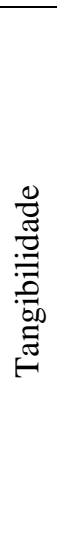 } & $\begin{array}{l}\text { A panificadora deveria dispor de estacionamento } \\
\text { acessível? }\end{array}$ & $\begin{array}{l}\text { A panificadora tem vagas disponíveis para } \\
\text { estacionar? }\end{array}$ \\
\hline 2 & & $\begin{array}{l}\text { As instalações físicas (iluminação, mesas e } \\
\text { expositores) deveriam ser visualmente atrativas e } \\
\text { estar em bom estado de conservação? }\end{array}$ & $\begin{array}{l}\text { As instalações físicas (iluminação, mesas e } \\
\text { expositores) são visualmente atrativas e estão } \\
\text { em bom estado de conservação? }\end{array}$ \\
\hline 3 & & $\begin{array}{l}\text { Os funcionários devem estar bem vestidos e } \\
\text { asseados (higiene pessoal)? }\end{array}$ & $\begin{array}{l}\text { Os funcionários estão bem vestidos e asseados } \\
\text { (higiene pessoal)? }\end{array}$ \\
\hline 4 & & $\begin{array}{l}\text { A panificadora deveria dispor de equipamentos } \\
\text { modernos? }\end{array}$ & $\begin{array}{l}\text { A panificadora dispõe de equipamentos } \\
\text { modernos? }\end{array}$ \\
\hline 5 & \multirow{5}{*}{ 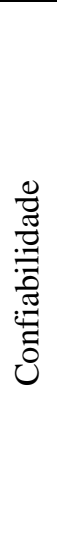 } & $\begin{array}{l}\text { O serviço prestado pela panificadora deve ser } \\
\text { realizado como prometido? }\end{array}$ & $\begin{array}{l}\text { O serviço prestado é realizado como } \\
\text { prometido? }\end{array}$ \\
\hline 6 & & & onfiança? \\
\hline 7 & & $\begin{array}{l}\text { A panificadora deveria fornecer o serviço no } \\
\text { tempo prometido? }\end{array}$ & $\begin{array}{l}\text { A panificadora fornece o serviço no tempo } \\
\text { prometido? }\end{array}$ \\
\hline 8 & & $\begin{array}{l}\text { A panificadora deveria oferecer uma variedade no } \\
\text { portfólio de produtos? }\end{array}$ & $\begin{array}{l}\text { A panificadora oferece uma variedade no } \\
\text { portfólio de produtos? }\end{array}$ \\
\hline 9 & & $\begin{array}{l}\text { A panificadora deveria identificar o tipo e o preço } \\
\text { dos produtos nos expositores? }\end{array}$ & $\begin{array}{l}\text { A panificadora identifica o tipo e o preço dos } \\
\text { produtos nos expositor? }\end{array}$ \\
\hline 10 & \multirow{4}{*}{ 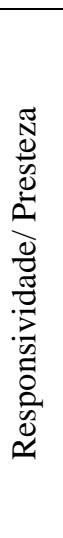 } & $\begin{array}{l}\text { A panificadora deve disponibilizar diferentes } \\
\text { formas de pagamento? }\end{array}$ & $\begin{array}{l}\text { A panificadora disponibiliza diferentes formas } \\
\text { de pagamento? }\end{array}$ \\
\hline 11 & & $\begin{array}{l}\text { A panificadora deveria ter disponibilidade em } \\
\text { solucionar os problemas dos clientes }\end{array}$ & $\begin{array}{l}\text { A panificadora mostra disponibilidade em } \\
\text { solucionar os problemas dos clientes? }\end{array}$ \\
\hline 12 & & $\begin{array}{l}\text { Quando os clientes tem algum problema com a } \\
\text { panificadora ela deveria ser solidária? }\end{array}$ & $\begin{array}{l}\text { Quando os clientes tem algum problema com } \\
\text { a panificadora ela resolver de maneira } \\
\text { solidária? }\end{array}$ \\
\hline 13 & & $\begin{array}{l}\text { A panificadora deveria ter rapidez e atenção em } \\
\text { atender os clientes? }\end{array}$ & $\begin{array}{l}\text { A panificadora tem rapidez e atenção em } \\
\text { atender os clientes? }\end{array}$ \\
\hline
\end{tabular}




\begin{tabular}{|c|c|c|c|}
\hline 14 & & $\begin{array}{l}\text { A panificadora deveria ter compromisso com os } \\
\text { clientes? }\end{array}$ & $\begin{array}{l}\text { A panificadora tem compromisso com os } \\
\text { clientes? }\end{array}$ \\
\hline 15 & & $\begin{array}{l}\text { Os funcionários da panificadora deveriam ter } \\
\text { conhecimento das suas funções específicas? }\end{array}$ & $\begin{array}{l}\text { Os funcionários da panificadora têm } \\
\text { conhecimento das suas funções específicas? }\end{array}$ \\
\hline 16 & 芯 & $\begin{array}{l}\text { Os funcionários devem realizar o atendimento } \\
\text { com eficiência (caixa, restaurante e padaria)? }\end{array}$ & $\begin{array}{l}\text { Os funcionários realizam o atendimento com } \\
\text { eficiência (caixa, restaurante e padaria)? }\end{array}$ \\
\hline 17 & & $\begin{array}{l}\text { Seus empregados deveriam obter suporte } \\
\text { adequado da empresa para cumprir suas tarefas } \\
\text { corretamente? }\end{array}$ & $\begin{array}{l}\text { Os empregados obtêm suporte adequado da } \\
\text { empresa para cumprir suas tarefas } \\
\text { corretamente? }\end{array}$ \\
\hline 18 & \multirow{5}{*}{ : } & $\begin{array}{l}\text { Os funcionários devem saber responder as } \\
\text { solicitações dos clientes com cortesia? }\end{array}$ & $\begin{array}{l}\text { Os funcionários sabem responder as } \\
\text { solicitações dos clientes com cortesia? }\end{array}$ \\
\hline 19 & & $\begin{array}{l}\text { Espera-se que os funcionários deem atenção } \\
\text { personalizada aos clientes? }\end{array}$ & $\begin{array}{l}\text { Os funcionários dão atenção personalizada aos } \\
\text { clientes? }\end{array}$ \\
\hline 20 & & $\begin{array}{l}\text { Os funcionários precisam conhecer as } \\
\text { necessidades dos seus clientes? }\end{array}$ & $\begin{array}{l}\text { Os funcionários conhecem as necessidades } \\
\text { dos seus clientes? }\end{array}$ \\
\hline 21 & & $\begin{array}{l}\text { É importante a panificadora ter como o objetivo a } \\
\text { satisfação dos clientes? }\end{array}$ & $\begin{array}{l}\text { A panificadora tem como objetivo a satisfação } \\
\text { dos clientes? }\end{array}$ \\
\hline 22 & & $\begin{array}{l}\text { O horário de funcionamento da panificadora } \\
\text { deveria ser conveniente para todos os clientes? }\end{array}$ & $\begin{array}{l}\text { A panificadora tem o horário de } \\
\text { funcionamento conveniente para todos os } \\
\text { clientes? }\end{array}$ \\
\hline
\end{tabular}

Fonte: Autoria Própria (2016)

(iii) Aplicação da escala SERVQUAL:

A aplicação dos questionarios se deu somente em horários determinados, das 09:00 às 10:00 horas e das 17:00 às 18:00 horas, horários de maior público. Os dados foram coletados sempre nesses horários e em todos os dias da semana, iniciando em uma segunda-feira e finalizando no domingo, para cada uma das panificadoras. Dessa forma, foi possivel padronizar as amostras em relação aos horários e dias da semana em que foram coletadas.

A equipe destinada a aplicação dos questionarios foi treinada, visando padronizar a coleta de dados. Estipulou-se que sempre o entrevistador, após terminar o preenchimento de um questionário, iria novamente para a porta do estabelecimento e aboradaria a primeira pessoa que entrasse para preencher o próximo questionário, fazendo uma escolha aleatória dos clientes.

No treinamento realizado, houve preecupação também em se padronizar as explicações fornecidas pelo entrevistador e em não induzir nenhum tipo de resposta em relação às questões propostas.

(iv) Análise dos resultados encontrados: 
Os dados obtidos foram organizdos em planilha do Excel para o cálculo das médias, desvios padroes, variâncias GAP e Alfa de Cronbach.

A validação dos dados obtidos foi caracterizada pela utilização do coeficiente Alfa de Cronbach, uma medida de confiabilidade do questionário aplicado, comumente utilizada para a avaliação da consistência interna dos questionários para um conjunto de dois ou mais indicadores de constructo (MATTHIENSEN, 2011). Onde os valores deste coeficiente variam de 0,0 a 1,0 e a maior proximidade do número 1,0 representa maior confiabilidade, sugerindo que o questionário está devidamente elaborado e pode ser considerado válido e confiável (ALMEIDA, SANTOS E COSTA, 2010).

O coeficiente Alfa de Cronbach foi calculado a partir do somatório das variâncias dos itens individuais e do somatório das variâncias de cada avaliador, conforme mostrado na Equação 3 (MATTHIENSEN, 2011).

$$
\alpha=\left(\frac{K}{K-1}\right)(1
$$

Onde:

$\mathrm{K}=$ corresponde ao número de itens (perguntas) do questionário;

$S^{2}{ }_{i}=$ corresponde à variância de cada item;

$S^{2}{ }_{t}=$ corresponde à variância total do questionário (somatório das variâncias dos avaliadores)

Após a análise da confiabilidade do questionário foi realizado a purificação dos dados. A purificação foi realizada para verificar o comportamento do coeficiente de Alfa de Cronbach e assim eliminar as questões que estejam prejudicando a confiabilidade do questionário. Para efetuar a purificação foi identificada a questão com maior variância registrada entre as 22 perguntas, em seguida, elimina-se essa pergunta e faz-se novamente os cálculos do coeficiente Alfa de Cronbach com dos dados purificados, sem a questão de variância elevada.

\section{Resultados e Discussões}

Nesta seção são apresentados e discutidos os resultados da aplicação do SERVQUAL, da análise da influência da faixa etária e da escolaridade nos GAP e da avaliação da confiabilidade do questionário proposto.

Ao analisar o comparativo dos GAP $=\mathrm{P}-\mathrm{E}$ das perguntas para cada panificadora na Figura 2, observou-se que a diferença entre percepção $(\mathrm{P})$ e expectativa (E) dos clientes quanto aos serviços oferecidos apresentaram em sua totalidade valores negativos, mostrando que os serviços prestados pelas panificadoras não atendiam as expectativas dos clientes.

A dimensão da qualidade tangibilidade apresentou os menores valores de GAP: - 3,4 ; - 3,0 e - 1,6, relacionadas à primeira pergunta do questionário que trata da acessibilidade do 
estacionamento (questão 1), respectivamente às panificadoras B, A e C, porém está questão também apresentou a maior variância relativa as respostas obtidas $\left(S^{2}{ }_{i}=4,31\right.$ para o desempenho e $S^{2}{ }_{i}=$ 1,01 para a expectativa), mostrando a discrepância das respostas dos entrevistados para essa questão.

Outras questões que também apresentaram valores consideravelmente baixos de GAP foram: para a panificadora A as questões 9 e 13 (valores de GAP respectivamente $-1,7$ e - 1,8) e para a panificadora B as questões 4; 9 e 17 (valores de GAP respectivamente - 1,6; - 1,6 e - 1,5). De forma geral, as panificadoras A e B apresentaram valores mais negativos para os GAP.

Figura 2: GAP calculado para cada uma das 22 questões.

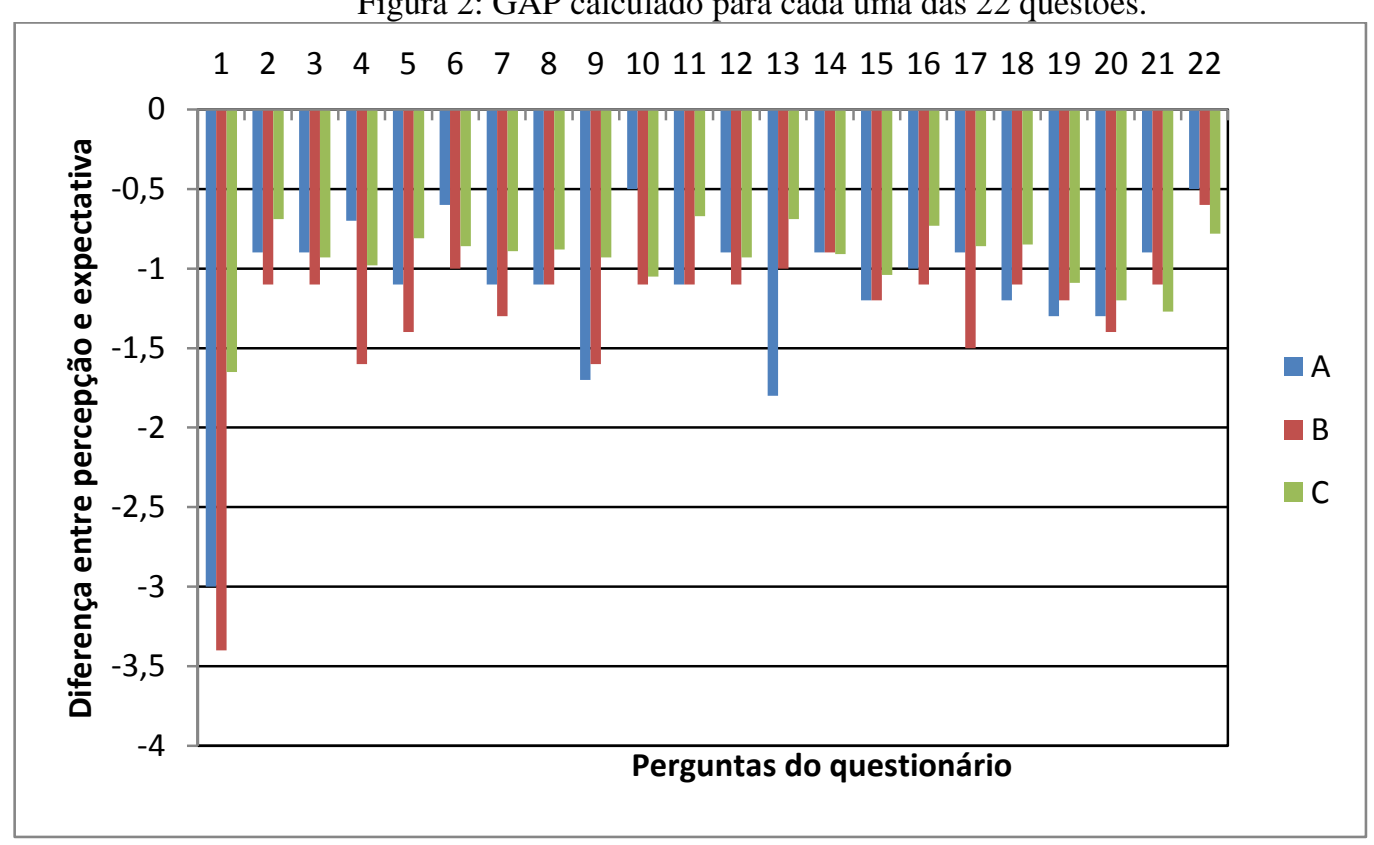

Fonte: Pesquisa de campo (2016)

O maior GAP $(-0,5)$ foi apresentado pela panificadora A para a dimensão da qualidade responsividade/presteza e empatia, referindo-se a disponibilização de diferentes formas de pagamento (Questão 10) e a conveniencia de horários de funcionamento (questão 22). 


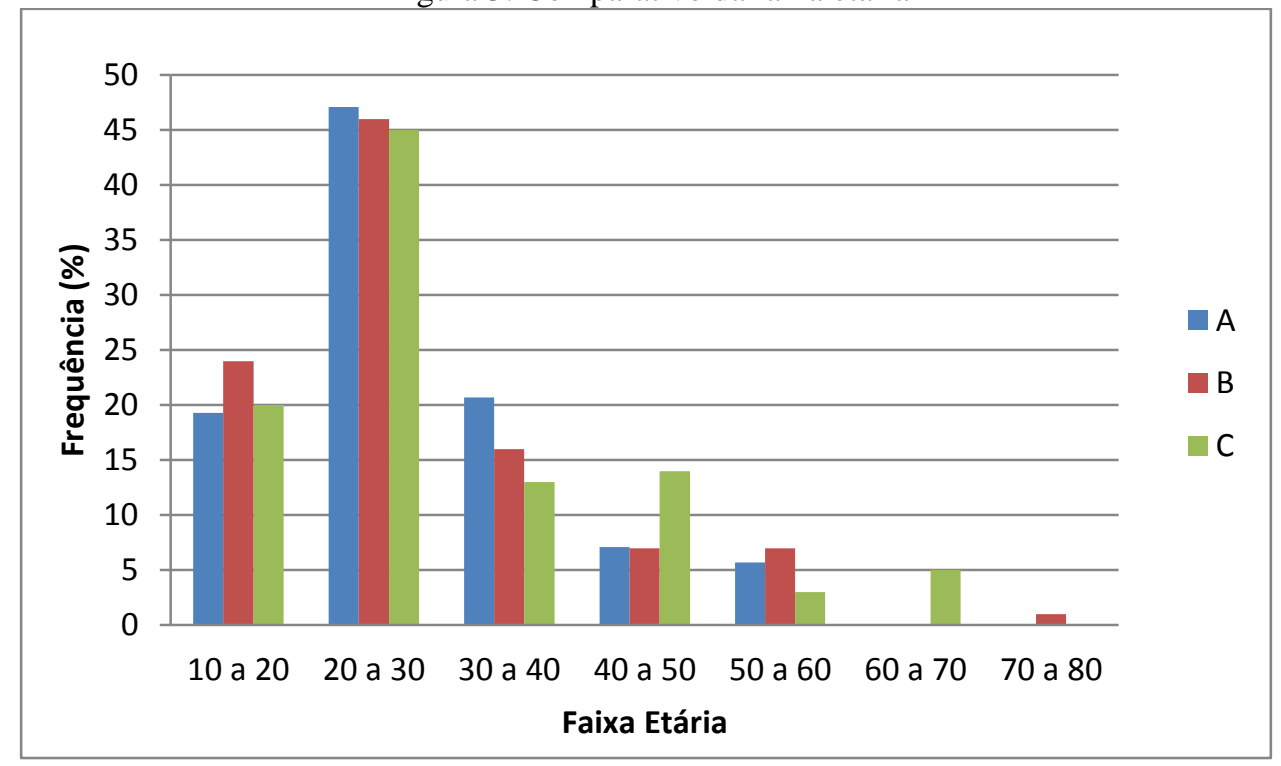

Fonte: Pesquisa de campo (2016)

Em relação à faixa etária dos clientes, a Figura 3 mostra que as panificadoras $\mathrm{A}$ e $\mathrm{B}$ apresentam maior público entre 10 a 40 anos, com $87 \%$ e $86 \%$ respectivamente, do que a panificadora $\mathrm{C}$, com $78 \%$. Pode-se identificar, portanto, que o público da panificadora $\mathrm{C}$ possui maior faixa etária quando comparado às outras duas panificadoras ( $22 \%$ dos clientes possuem mais de 40 anos).

Para o nível de escolaridade, a Figura 4 mostra que $42 \%$ do público da panificadora $\mathrm{C}$ possui escolaridade até o Ensino Médio Incompleto, diferente das panificadoras A e B em que essa porcentagem fica em $19 \%$ e $13 \%$, respectivamente. Enquanto a porcentagem de clientes que possui Ensino Superior Incompleto, Ensino Superior ou Técnico Completo e Incompleto é de 51\% para A, $45 \%$ para B e $23 \%$ para $\mathrm{C}$.

Os analfabetos não foram contabilizados no estudo, pois não houve nenhuma entrevista realizada com eles, possivelmente por não frequentarem o horário no qual se aplicou o questionário ou porque se recusaram a contribuir com a pesquisa. 
Figura 4: Comparativo da escolaridade entre as panificadoras.

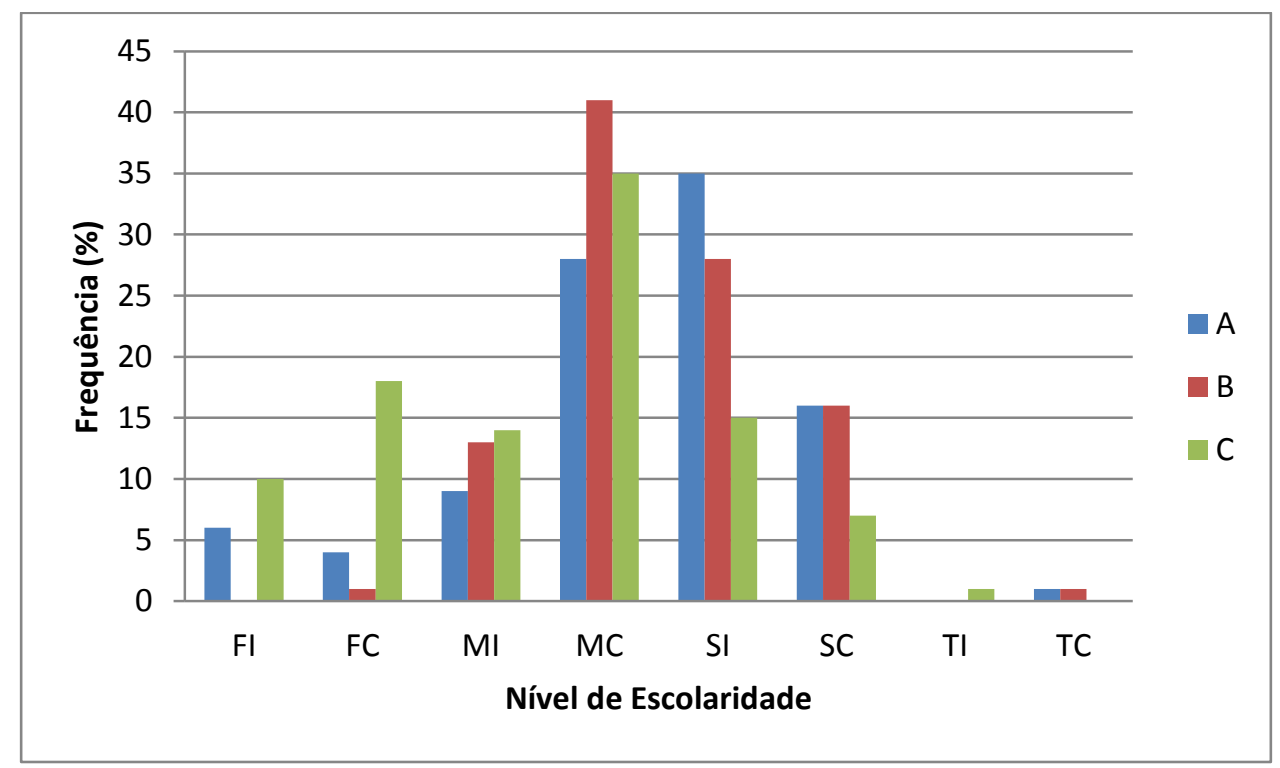

Legenda: FI: Fundamental Incompleto, FC: Fundamental Completo, MI: Médio Incompleto, MC: Médio Completo, SI: Superior Incompleto, SC: Superior Completo, TI: Técnico Incompleto, TC: Técnico Completo.

Fonte: Pesquisa de campo (2016)

O estudo da validade e confiabilidade do questionário proposto realizado pelo coeficiente Alfa de Cronbach apresentou valores próximos a 1,0, ou seja, apresentou uma boa confiabilidade.

No estudo da validade do desempenho, a variância de cada item $\left(\mathrm{S}_{\mathrm{i}}^{2}\right)$ ficou entre 1,2471 (para a questão 16) e 4,3126 (para a questão 1). Somente seis questões apresentaram $S_{i}^{2}>2,0$ enquanto que a primeira questão teve variância significativamente grande $\left(S_{i}^{2}=4,3126\right)$ e média em torno de 3,8678 .

Para confiabilidade dos resultados em relação à expectativa dos clientes, a variância para cada item $\left(\mathrm{S}^{2}{ }_{\mathrm{i}}\right.$ ) ficou entre 1,0138 (questão 1) e 0,1781 (questão 16). Com exceção da primeira questão que apresentou valor maior que 1,0, todas as demais perguntas apresentaram valores abaixo deste valor. A média para a primeira questão foi 6,5552 .

O somatório das variâncias $S^{2}$ mostra uma maior discrepância nas respostas relativas ao desempenho $\left(\Sigma \mathrm{S}_{\mathrm{i}}{ }^{2}=40,4497\right)$, indicando que os entrevistados possuem expectativas parecidas relativas à qualidade do serviço $\left(\Sigma \mathrm{S}_{\mathrm{i}}{ }^{2}=11,5095\right)$, porém apresentam maior discordância em relação às suas percepções sobre a qualidade do serviço prestado.

Para as duas classes, desempenho e expectativa, houve uma grande dispersão dos resultados em relação à questão 1 apenas, mostrando que os entrevistados apresentaram grande diferença nas respostas somente para essa questão.

Os resultados da avaliação da confiabilidade de acordo com o coeficiente Alfa de Cronbach são mostrados na Tabela 2, apresentando valores próximos a 1,0 $\left(\alpha_{\mathrm{des}}=0,9800\right.$ e $\alpha_{\mathrm{exp}}=0,9750$, para desempenho e expectativa respectivamente) indicando alto grau de confiabilidade dos questionários. 
Tabela 2: Resultados dos cálculos de Alfa de Cronbach.

\begin{tabular}{l|cc}
\hline & DESEMPENHO & EXPECTATIVA \\
\hline $\mathrm{A}$ & 0,9800 & 0,9750 \\
$\mathrm{~K}$ & 22 & 22 \\
$\Sigma \mathrm{S}_{\mathrm{i}}{ }^{2}$ & 40,4497 & 11,5095 \\
$\Sigma \mathrm{S}_{\mathrm{t}}{ }^{2}$ & 628,3654 & 166,6264 \\
\hline \multicolumn{2}{c}{} \\
\hline \multicolumn{2}{|c}{ Fonte: Autoria própria (2016) }
\end{tabular}

Na Tabela 2, K é o número de perguntas tanto das perspectivas quanto das expectativas e $\alpha$ é o coeficiente Alfa de Cronbach.

Fazendo-se a purificação do questionário, eliminando-se o item com alta variância, nesse caso, a questão 1, obtém-se um grau ainda maior de confiabilidade do questionário, $\alpha_{\text {des }}=0,9800 \mathrm{e}$ $\alpha_{\exp }=0,9900$, respectivamente para o desempenho e para a expectativa. Observa-se que não houve alteração para o Alfa de Cronbach do desempenho, porém a purificação teve resultado para a expectativa, mostrando, para esse caso, que as discrepâncias das expectativas relacionadas à questão 1 afetam o Alfa de Cronbach.

A análise dos 416 questionários das três panificadoras mostrou as diferenças entre a faixa etária e a escolaridade dos clientes e a influência desses parâmetros nas suas expectativas e percepções a respeito da qualidade do serviço prestado.

$\mathrm{O}$ público da panificadora $\mathrm{C}$ é diferenciado em relação às outras duas, possui menor escolaridade e maior faixa etaria, o que influencia significativamente na percepção da qualidade, pois apesar panificadora da $\mathrm{C}$ possuir estrutura mais modesta ela apresentou de uma forma geral os maiores GAP's, ou seja, menor diferença entre o que é esperado pelos clientes e o que é percebido por eles. Portanto, a avaliação da qualidade mensurada pelo SERVQUAL é influenciada significativamente pelo perfil do público alvo.

\section{Conclusões}

A aplicação do modelo SERVQUAL nos estabelecimentos de panificação possibilitou compreender sob a ótica do cliente, a forma como estes avaliam a qualidade dos serviços, por meio das diferenças das médias entre as percepções e expectativas em relação ao serviço prestado.

A dimensão da qualidade tangibilidade, especificamente em relação à acessibilidade no estacionamento, chamou a atenção por apresentar GAP bastante baixo, mostrando a importância dada e as dificuldades apresentadas pelos clientes em relação a essa questão. 
Esse trabalho vem demostrar a influência do perfil dos clientes na avaliação da qualidade nos serviços prestados. É notória a diferença dos clientes da panificadora $\mathrm{C}$ em relação aos estabelecimentos A e B, em que o público daquela possui menor escolaridade e maior faixa etária em relação às outras duas, características indicadoras de diferenças nos critérios de avaliação da qualidade nos serviços, pois a empresa $\mathrm{C}$ apresentou os maiores valores dos GAP em relação às percepções e expectativas, mostrando que seus clientes possuem maior satisfação em relação aos serviços prestados em comparação às empresas A e B.

Esta assertiva é corroborada com o fato das panificadoras A e B apresentarem estrutura mais moderna e mesmo assim apresentarem menores GAP nas questões relacionadas, mostrando que seus clientes possuem menor satisfação em relação aos serviços prestados em comparação ao estabelecimento C. Desta forma, fica evidente que públicos com diferentes escolaridade e faixa etária apresentam distintas percepções da qualidade, determinando os resultados da avaliação da qualidade nos serviços feita pelo SERVQUAL.

Espera-se que esse estudo possa auxiliar na forma de interpretação dos resultados do SERVQUAL, salientando a influência do perfil do cliente na avaliação da qualidade, contribuir com a percepção da relevância da qualidade em serviços e também proporcionar mudanças efetivas em relação à melhoria dos serviços prestados.

A partir da análise da confiabilidade dos questionários, verificou-se que o questionário proposto mostrou-se adequado para o estudo realizado, pois o coeficiente Alfa de Cronbach apresentou valores indicando boa confiabilidade.

\section{Abstract}

The work deals the analysis and quantification of customer satisfaction three bakeries of ItacoatiaraAM to the quality of services provided, identifying the dimensions of quality and the main problems in the customer view. The tool used for the application of planning, analysis and processing of data was the SERVQUAL, first proposed by Parasuraman, Zeithaml and Berry. For this, we applied a questionnaire with 24 questions (22 parameters of quality and two questions about age and education) into random samples of 416 questionnaires filled out by customers of bakeries. In addition to the application of SERVQUAL also considered whether to influence the parameters of quality in services in terms of age and customer education level. This work allowed us to analyze the GAP between the perceptions and expectations of customers, among which the quality dimension tangibility (parking accessibility) had the lowest value, for exemple, greater customer dissatisfaction regarding expected in service. One could also identify significant differences in GAP to age and education of clients of the three bakeries. For the questionnaire reliability analysis was used Cronbach alpha coefficient. The study also identified the critical points related to the quality of services and allowed to discuss the influence of the customer profile the results with the application of SERVQUAL, allowing proposing improvements to the projects through more detailed analyzes of the results.

Key-words: SERVQUAL; quality services; breadmaker. 


\section{Referências}

ALMEIDA, D.;SANTOS,M.C.R; COSTA, A. F. B. Aplicação do coeficiente alfa de cronbach nos resultados de um questionário para avaliação de desempenho da saúde pública. São Carlos: XXX Encontro Nacional de Engenharia de Produção, 2010.

BARRETO, E. G. L; SANTOS, R.L. S.; SILVA, L.B.; GOMES, M. L. Barreto; MENEZES, V. L. Aplicação do método servqual na avaliação da satisfação de clientes de uma academia de ginástica. Universidade Tecnológica Federal do Paraná. Revista Gestão Industrial, Paraná, v. 08. n. 03: p. 91-108, 2012.

BATALHA, Mario Otávio. Introdução á Engenharia de Produção. Rio de Janeiro: Elsevier, 2008.

CHASE, Richard B. Administração da produção para a vantagem competitiva. Porto Alegre: Bookman, 2006.

GONÇAlVES, J. E. L; OLIVEIRA, V. L. Atendimento ao Público na Prestação de Serviços. Ano 2, No 5 . Rio de Janeiro: CADERNOS FGV PROJETOS, 2007.

GUARIENTI, A.; POLL, M. T.; BRESOLIN, R. R.; GODOY, L. P. Avaliação da Qualidade percebida em Serviços através da Escala SERVQUAL, 2006. Trabalho apresentado XXVI Encontro Nacional de Engenharia de Produção, Fortaleza, 2006. Disponível em: < http://www.abepro.org.br/biblioteca/ ENEGEP2006_TR470326_7203.pdf>. Acesso em: 14 de Outubro de 2013.

Instituto Brasileiro de Geografia e Estatística, 2013. Infográficos: despesas e receitas orçamentárias e PIB. Disponível em:

<http://cidades.ibge.gov.br/painel/economia.php?lang=\&codmun=130190\&search=amazonas|itacoatiara|infograficos:despesas-e-receitas-orcamentarias-e-pib>. Acesso em: 14 de Outubro de 2013.

JOHNSTON, R., CLARK, G. Administração de Operações de Serviço. São Paulo: Atlas, 2009.

LACERDA, D. A.; OLIVEIRA, J. M; WISLER, M.. Qualidade nos serviços prestados por um hotel de selva no município de Itacoatiara/AM, segundo a ótica do turista. Bauru: XX Simpósio de Engenharia de Produção, 2013.

LOVELOCK, C; WRIGHT, L. Serviços: Marketing e Gestão. São Paulo: Saraiva, 2001.

MATTHIENSEN, Alexandre. Uso do Coeficiente Alfa de Cronbach em Avaliações por Questionários. Embrapa Roraima: Boa Vista, 2011.

PELISSARI, A. S.; SOLIS, D. R.; IANAGUI, E. C.; GONZALEZ, I. V. D. P; SETUBAL, Flávia Meneguelli Ribeiro. Aplicação e Avaliação do Modelo Servqual para Analisar a Qualidade do Serviço. Trabalho apresentado VIII Simpósio de Excelência em Gestão e Tecnologia, 2011. Disponível em: <http://www.aedb.br/seget/artigos11/1_Artigo\%20Aplicacao\%20e\%20Avaliacao\%20do\%20Modelo\%20Servqual\%20p ara\%20Analisar\%20a\%20Qualidade\%20do\%20Servico.pdf >. Acesso em 17 de Outubro de 2013.

PRASS, R. M.; SANT'ANNA, L. C.; GODOY, L. P. Avaliação da Qualidade de Serviços Prestados na Área Educacional através do Modelo SERVQUAL. Paraná: Revista Gestão Industrial, v. 06, n. 02: p. 213-231, 2010. crossref

PRASS, R. M.; SANT'ANNA, L. C.; GODOY, L. P. APUD LIMA, M.; SAPIRO, A. Vilhena, J. B.; GANGANA, M. Gestão de marketing. 3.ed. Rio de Janeiro, FGV, 2004.

Programa de Desenvolvimento da Alimentação, Confeitaria e Panificação (PROPAN). Perfil da Panificação. Disponível em: <http://www.propan.com.br/institucional.php?idcat=9>. Acesso em: 21 de Outubro de 2013.

SILVA, Luan Carlos Santos; KOVALESKI, João Luiz; GAIA, Silvia. Qualidade em serviços: uma análise teórica sobre as principais características. Revista Gestão Industrial: Paraná, 2011.

SLACK, Nigel; CHAMBERS, Stuart; JOHNSTON, Robert; BETTS, Alan. Gerenciamento de operações e de processos: Princípios e práticas de impacto estratégico. Porto Alegre: Bookman, 2008. 


\section{Dados dos autores:}

\section{Autora: Luana Moraes da Silva}

Filiação Institucional: Universidade Federal do Amazonas

Cargo: Estudante

Telefone: (92) 993754394

E-mail: luanamoraes.ep@gmail.com

\section{Autor: Rodrigo Bíscaro Nogueira}

Filiação Institucional: Universidade Federal do Amazonas

Cargo: Professor

E-mail: rodrigobiscaro@gmail.com

\section{Autor: João Marcos de Oliveira}

Filiação Institucional: Universidade Federal do Amazonas

Cargo: Professor

E-mail:jmoprofe@gmail.com

\section{Autor: Christiano Luna Arraes}

Filiação Institucional: Universidade Federal do Amazonas

Cargo: Professor

E-mail: chistianoarraes@yahoo.com.br

Submetido em: 24-02-2016

Aceito em: 15-06-2016 